\title{
Mirror mode waves in Venus's magnetosheath: solar minimum vs. solar maximum
}

\author{
Martin Volwerk $^{1}$, Daniel Schmid ${ }^{1,2}$, Bruce T. Tsurutani ${ }^{3}$, Magda Delva $^{1}$, Ferdinand Plaschke $^{1}$, Yasuhito Narita ${ }^{1}$, \\ Tielong Zhang ${ }^{1,4}$, and Karl-Heinz Glassmeier ${ }^{5}$ \\ ${ }^{1}$ Space Research Institute, Austrian Academy of Sciences, Graz, Austria \\ ${ }^{2}$ University of Graz, NAWI Graz, Graz, Austria \\ ${ }^{3}$ California Institute of Technology, Pasadena, California, USA \\ ${ }^{4}$ CAS Key Laboratory of Geospace Environment, University of Science and Technology of China, Hefei, China \\ ${ }^{5}$ Institute for Geophysics and Extraterrestrial Physics, TU Braunschweig, Braunschweig, Germany \\ Correspondence to: Martin Volwerk (martin.volwerk@oeaw.ac.at)
}

Received: 30 May 2016 - Revised: 29 September 2016 - Accepted: 8 November 2016 - Published: 23 November 2016

\begin{abstract}
The observational rate of mirror mode waves in Venus's magnetosheath for solar maximum conditions is studied and compared with previous results for solar minimum conditions. It is found that the number of mirror mode events is approximately $14 \%$ higher for solar maximum than for solar minimum. A possible cause is the increase in solar UV radiation, ionizing more neutrals from Venus's exosphere and the outward displacement of the bow shock during solar maximum. Also, the solar wind properties (speed, density) differ for solar minimum and maximum. The maximum observational rate, however, over Venus's magnetosheath remains almost the same, with only differences in the distribution along the flow line. This may be caused by the interplay of a decreasing solar wind density and a slightly higher solar wind velocity for this solar maximum. The distribution of strengths of the mirror mode waves is shown to be exponentially falling off, with (almost) the same coefficient for solar maximum and minimum. The plasma conditions in Venus's magnetosheath are different for solar minimum as compared to solar maximum. For solar minimum, mirror mode waves are created directly behind where the bow shock will decay, whereas for solar maximum all created mirror modes can grow.
\end{abstract}

Keywords. Magnetospheric physics (magnetosheath; plasma waves and instabilities) - space plasma physics (waves and instabilities)

\section{Introduction}

Mirror mode (MM) waves are a key ingredient of the wave activity in planetary and cometary magnetosheaths (see, e.g. Tsurutani et al., 1982; Erdös and Balogh, 1993; Glassmeier et al., 1993; Bavassano Cattaneo et al., 1998; Baumjohann et al., 1999; Lucek et al., 1999; Joy et al., 2006; Schmid et al., 2014; Volwerk et al., 2014; Soucek et al., 2015). The waves are generated by a temperature asymmetry and Hasegawa (1969) showed that for a bi-Maxwellian plasma the instability criterion is given by

$1+\beta_{\perp}\left(1-\frac{T_{\perp}}{T_{\|}}\right)<0$.

The newly created ions (from ionization of exospheric atoms) are picked up by the solar wind magnetic field creating a ring-beam distribution. Such distributions are unstable and will produce ion cyclotron waves or MM waves (see, e.g. Gary, 1991). At crossing the quasi-perpendicular bow shock the ions are mainly heated in the perpendicular direction, with respect to the background magnetic field, when compared to the parallel direction, increasing the already existing temperature asymmetry of the ring-beam distribution. Theoretically, the growth rate for MM waves was estimated by Gary (1991) to be proportional to the proton cyclotron frequency $\gamma \propto 0.1 \omega_{\mathrm{c}, \mathrm{p}}$; however, Tátrallyay et al. (2008) with spacecraft observations have shown that this is an overestimation.

Another driver for MM waves is magnetic field line draping (see also Tsurutani et al., 2011; Volwerk et al., 2008b), 
which serves as source of "free energy" in planetary magnetosheaths. As the shocked solar wind moves deeper into the magnetosheath, the planet will act as a conducting obstacle in the flow and will "hang up" the magnetic field in its neighbourhood, whereas the parts of the field lines further out from the Venus-Sun line will continue to flow with the magnetosheath flow velocity. This causes the field lines to drape around the planet (Zhang et al., 2010; Du et al., 2013). Field line draping around Venus's ionosphere has two effects: first it leads to a squeezing of the plasma, by which the hot$T_{\|}$plasma is sent towards the downstream region, and secondly the magnetic tension leads to an increase of $T_{\perp}$ (see also Crooker and Siscoe, 1977).

In the solar wind this distribution will generate mainly ion cyclotron waves (Delva et al., 2008) because of the solar wind plasma- $\beta$ usually being lower than 1 , but it sometimes also gives rise to MM waves at a very low occurrence rate of $\sim 4$ per day (Zhang et al., 2008b). In the magnetosheath, however, MM waves are most likely expected to be generated. Tsurutani et al. (2002) and Remya et al. (2014), however, showed that during a period of exceptionally low solar wind plasma- $\beta(\sim 0.35)$, the magnetosheath can be prone to a high occurrence rate of ion cyclotron waves (see also Czaykowska et al., 2001).

At Venus these MM waves were first discovered (Volwerk et al., 2008a) from the Venus Express mission (VEX, Svedhem et al., 2007) using only the magnetometer data (Zhang et al., 2006). The waves were shown to have a period between $\sim 4$ and $\sim 15 \mathrm{~s}$ depending on the location in the magnetosheath. A statistical study over 1 Venus year (i.e. 224 Earth days) during solar minimum was performed by Volwerk et al. (2008b), which showed that the occurrence rate of MM waves is highest just behind the bow shock as well as close to the ionopause: the former location because of the perpendicular heating by the bow shock increasing the temperature anisotropy and the latter location because of the magnetic field pile-up, increasing field strength and thereby the temperature anisotropy through the first adiabatic invariant. It was also demonstrated that MM waves are mainly generated for quasi-perpendicular bow shock conditions, as expected.

In this paper, the solar maximum data are analysed first to obtain the occurrence rate and strengths of the MM waves. Then the results are compared to those for solar minimum. Further statistical analysis is performed on the MM strength, and the growth rate is estimated for both solar conditions. A discussion about the differences and similarities between the two states of solar activity is then performed and the paper ends with some conclusions and concluding remarks.
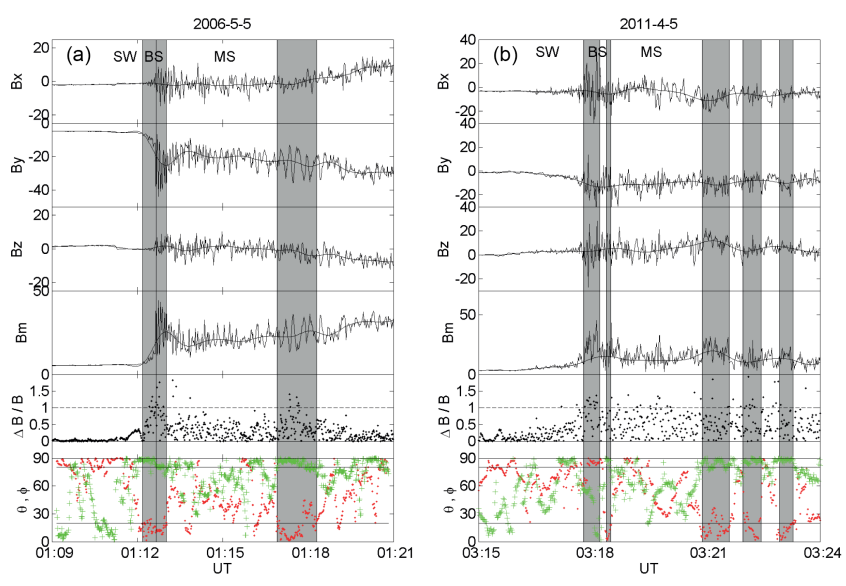

Figure 1. Bow shock crossing for solar minimum (left) and solar maximum (right). From top to bottom the three components and the amplitude of the magnetic field are shown, with the low-pass filtered data overplotted. The strength $\mathcal{B}$ and the two angles $\theta_{\mathrm{Bmv}}$ (red dots) and $\phi_{\mathrm{Bmv}}$ (green pluses) are in the bottom two panels. The grey shaded areas show location of MM intervals, except for the two labelled BS (bow shock).

\section{Two selected events}

In Fig. 1 two magnetosheath crossings are shown for solar minimum and solar maximum conditions. The solar minimum event has already been discussed by Volwerk et al. (2008a). It shows a quasi-perpendicular bow shock crossing with MM waves just behind the shock with a period of $\sim 5 \mathrm{~s}$ and closer to Venus an interval with $\mathrm{MM}$ waves with a period of $\sim 15 \mathrm{~s}$. The solar maximum event shows a more quasi-parallel bow shock crossing, which clearly has little MM activity directly behind the bow shock, but further in the magnetosheath there are intervals of MM waves with a period of $\sim 7 \mathrm{~s}$.

The magnetometer data are analysed using the method described by Lucek et al. (1999). For each $24 \mathrm{~h}$ orbit of VEX the time period that the spacecraft is near or inside Venus's bow shock is selected (see also Volwerk et al., 2008b). Over a full Venus year of 224 days this results in the following total amount of time that VEX was in Venus's magnetosheath: solar minimum $\sim 316 \mathrm{~h}$ and solar maximum $\sim 292 \mathrm{~h}$. The difference between these two values is because of slight orbital changes of VEX over the 5-year interval between solar minimum and maximum. A $20 \mathrm{~s}$ wide sliding-window minimum variance analysis is performed, with a shift of $1 \mathrm{~s}$. In order to identify MM waves the angles between the maximum $\left(\theta_{\mathrm{Bmv}}\right)$ and minimum $\left(\phi_{\mathrm{Bmv}}\right)$ variance direction and the background magnetic field are determined. For each point in the sliding window the strength

$\mathcal{B}=\Delta B / B=2\left|B-B_{\mathrm{bk}}\right| / B_{\mathrm{bk}}$

is determined, where $B$ is the magnetic field magnitude and the background magnetic field $B_{\mathrm{bk}}$ is obtained by low-pass 
filtering the data, where variations with periods shorter than 3 min are filtered out. In this paper the MM wave trains are called "events", where an event consists of sequential $20 \mathrm{~s}$ sliding windows in which the identification criteria are met and the smallest size of one event is one window. The MM identification criteria are as follows:

- a small angle $\theta_{\mathrm{Bmv}} \leq 20^{\circ}$ between maximum variance direction and background magnetic field (Price et al., 1986)

- a large angle $\phi_{\mathrm{Bmv}} \geq 80^{\circ}$ between minimum variance direction and background magnetic field

- a minimal strength during an event $\mathcal{B}>0.2$.

In Fig. 1 the two angles are shown as red dots $(\theta)$ and green pluses $(\phi)$, respectively. The grey-shaded intervals show MM waves, except for the two marked with BS (c), which indicate the bow shock.

\section{Bow shock location}

In Zhang et al. (2008a) the statistical location of the bow shock was determined using the observed crossings of the bow shock into and out of the magnetosheath. The equation for the conic section that was used to describe the bow shock is

$R_{\mathrm{BS}}=\frac{L}{1+\epsilon \cos (\mathrm{SZA})}$,

where $L$ is the terminator crossing, $\epsilon$ is the eccentricity and SZA is the solar zenith angle. Fitting the observed bow shock locations for $20^{\circ} \leq \mathrm{SZA} \leq 120^{\circ}$, Zhang et al. (2008a) found that $L \approx 2.14$ and $\epsilon \approx 0.621$, which leads to a terminator distance of $R_{\mathrm{BS}, \mathrm{t}} \approx 2.14 R_{\mathrm{V}}$, which is slightly smaller than the value $R_{\mathrm{BS}, \mathrm{t}} \approx 2.40 R_{\mathrm{V}}$ which was found by Russell et al. (1988) for solar maximum conditions using Pioneer Venus Orbiter data, with an eccentricity $\epsilon \approx 0.609$.

\section{Statistical study}

In order to extend the solar minimum statistical MM study (24 May-31 December 2006, Volwerk et al., 2008b) to solar maximum, 1 Venus year (224 Earth days) of the $1 \mathrm{~Hz}$ magnetometer data from Venus Express, around solar maximum 2011-2012, was used (1 November 2011-10 June 2012) and processed in the same way. The MM waves that were found in Venus's magnetosheath are shown in Fig. 2 in cylindrical coordinates $X_{\mathrm{VSO}}$, and the distance of VEX from the VenusSun line $R=\sqrt{Y_{\mathrm{VSO}}^{2}+Z_{\mathrm{VSO}}^{2}}$.

First, there are more events for solar maximum (a total of 1857 events) than for solar minimum (a total of 1637 events). Also, it can be seen that the events for solar maximum already appear more distant from Venus as the nominal solar maximum bow shock location (Russell et al., 1988) is at
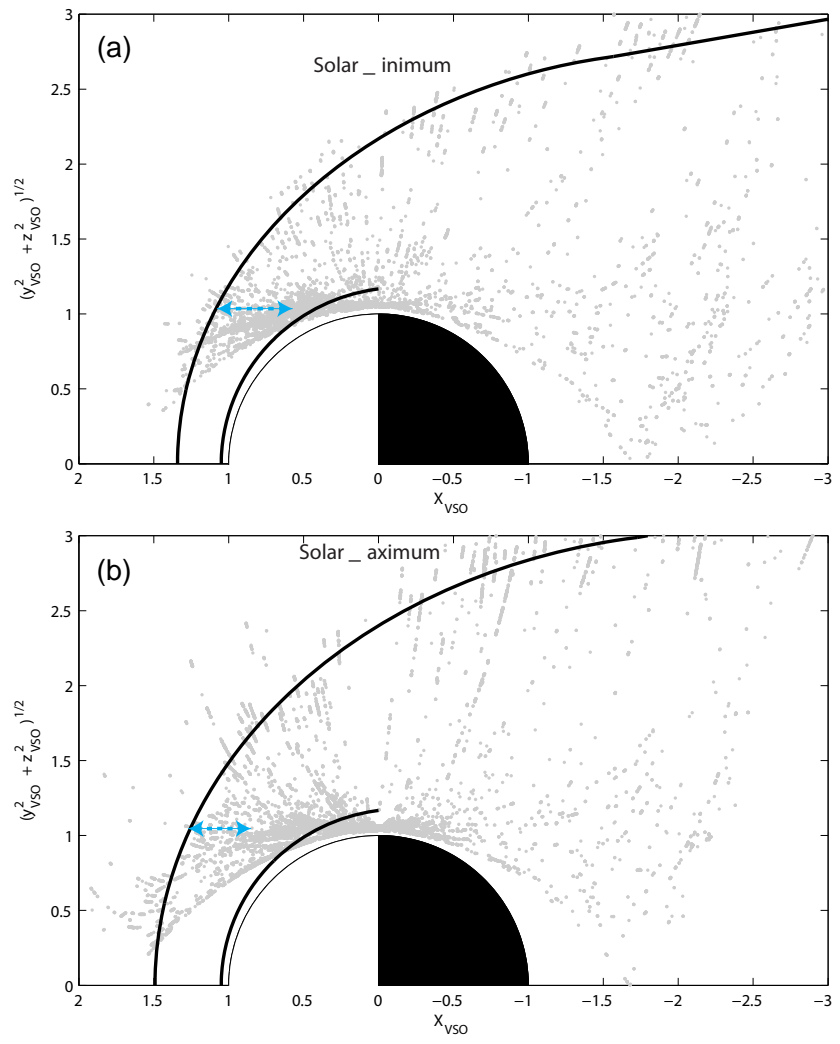

Figure 2. Positions of the MM events found in the magnetosheath for solar minimum (top, a total of 1637 events) and maximum (bottom, a total of 1857 events). The two thick black curves in each panel show the location of the bow shock as taken from Zhang et al. (2008a) for solar minimum and from Russell et al. (1988) for solar maximum. The solid line close to Venus is the location of the ionosphere, taken from Zhang et al. (2008a) in both cases. The cyan dashed arrows in both panels show the distance along the flow line of one event to the model bow shock.

greater distances than the solar minimum bow shock location (Zhang et al., 2008a).

For solar maximum the ionization rate around Venus is much higher than for solar minimum (see also, e.g. Delva et al., 2015), and thus the bow shock and ionopause move outward from Venus (e.g. Alexander and Russell, 1985; Shan et al., 2015).

\subsection{Mirror mode wave observation rate}

Using the location of $\mathrm{VEX}$ and the time interval that the spacecraft is within a $0.25 \times 0.25 R_{\mathrm{V}}$ box, the MM observation rate is calculated, defined as

$P=\frac{\text { number of events in box }}{\text { time spent in box }}$.

Although there are, as expected, differences in the details of the observation rate plots in Fig. 3, basically the major differences are the higher number of events for solar maximum 
(a)

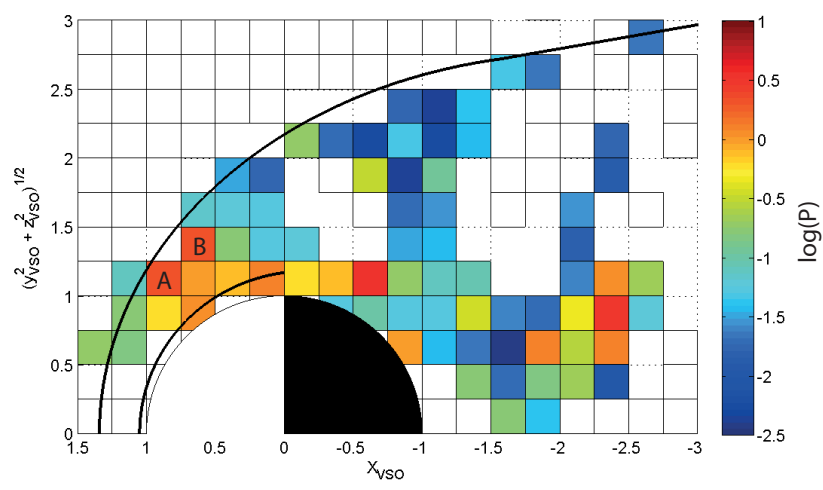

(b)

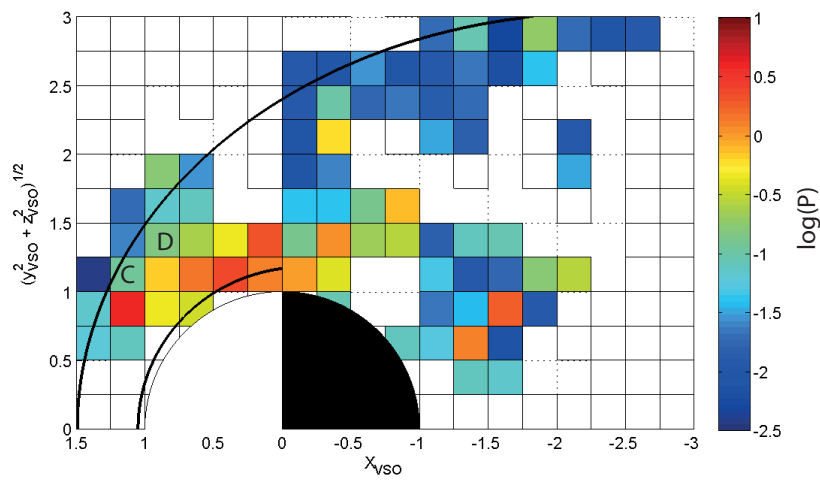

Figure 3. Comparison of the observational rate $P$ of MM waves in Venus's magnetosheath for solar minimum (top) and solar maximum (bottom). The two thick black curves in each panel show the location of the model ionosphere and model bow shock as in Fig. 2.

(1857 vs. 1637) and more events further away from Venus as the bow shock location moves outward. The highest observation rates can be found behind the bow shock and towards the ionopause, along the flow channel of the plasma in the magnetosheath, parallel to the $X_{\mathrm{VSO}}$ axis, close to the Venus-Sun line with $1.0 \leq R \leq 1.5$. This will be discussed in more detail below. As a comparison, the maximum observational rate for solar minimum is $P \approx 3$ events per hour, whereas for solar maximum the maximum observational rate is $P \approx 4$ events per hour.

Taking a closer look at the two panels in Fig. 3 and the distribution of the observational rate, a different behaviour for solar minimum and maximum can be seen. Whereas for solar minimum the highest rates are observed close to the nominal bow shock (e.g. the red squares labelled A and B) and then decrease deeper inside the magnetosheath, for solar maximum the rates are low behind the nominal bow shock (e.g. the greenish squares labelled $\mathrm{C}$ and $\mathrm{D}$ ) and the rate increases along the magnetosheath. This indicates a different
Table 1. The results of the slopes $a$ of the linear fits to the weak and strong MM waves in Fig. 4.

\begin{tabular}{lrrrr}
\hline & Weak $a$ & $\chi^{2}$ & Strong $a$ & $\chi^{2}$ \\
\hline Solar minimum & $-3.39 \pm 0.02$ & 0.99 & $-2.45 \pm 0.10$ & 0.89 \\
Solar maximum & $-3.04 \pm 0.03$ & 0.98 & $-1.82 \pm 0.10$ & 0.87 \\
\hline
\end{tabular}

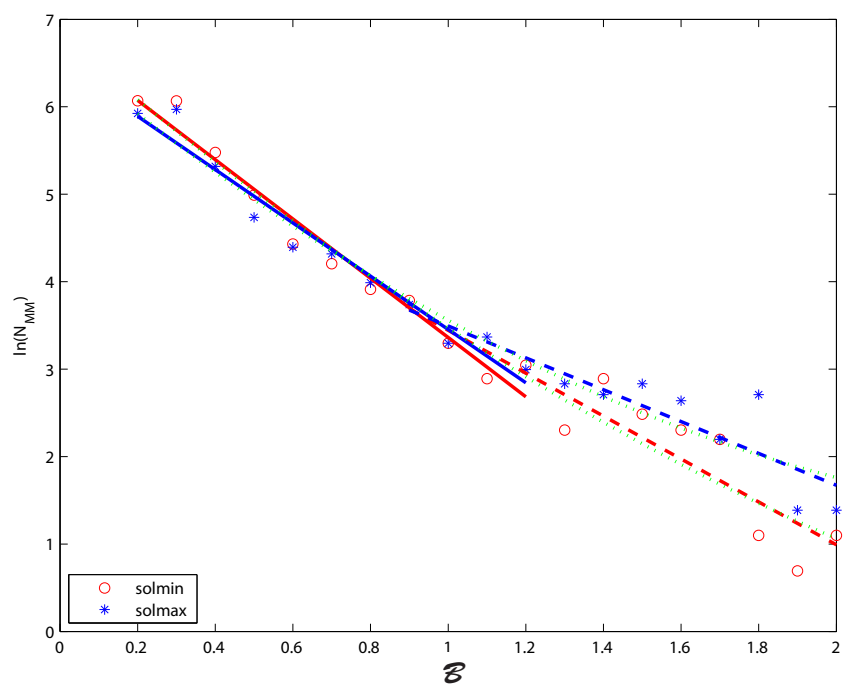

Figure 4. The $M M$ waves binned by $\mathcal{B}$, for 0.1 -size bins, for solar minimum (red circles) and maximum (blue asterisks). The solid (dashed) lines show exponential fits to the points for weak (strong) MM waves, with the fit parameters displayed in Table 1. The dotted green lines show second-order polynomial fits to all points.

growth rate for solar minimum and maximum, which will be discussed in Sect. 4.3.

\subsection{Mirror mode wave strength}

To investigate the distribution of strengths $\mathcal{B}$ of the $\mathrm{MM}$ waves, the events are binned with a bin size of $\Delta \mathcal{B}=0.1$. The results for both solar minimum and maximum are shown in Fig. 4 by red circles and blue asterisks respectively (see also Fig. 3 in Volwerk et al., 2008b). For both distributions a second-order polynomial has been fitted and is shown as a grey dotted line, which indicates a change of slope. Therefore, the weak $(\mathcal{B} \leq 1.2)$ and strong $(\mathcal{B} \geq 0.8)$ events are also fitted linearly:

$\log \left(N_{\mathrm{mm}}(\mathcal{B})\right) \propto a \cdot \mathcal{B}$

and they are shown as a solid and dashed line respectively. The results of this linear fit are shown in Table 1.

For the weak part, $\mathcal{B} \leq 1.2$, the slopes are quite similar; however, for the strong events there is a larger difference in the slopes. These fit values probably reflect the (varying) growth rate of the MM waves, which will be discussed in the following section. 
Table 2. Growth rates of MM waves $\left(s^{-1}\right)$ for solar minimum and maximum as determined for various quantities of the event distribution shown in Fig. 6 for pre- $\left(-0.25 \leq X_{\mathrm{VSO}} \leq 0.75\right)$ and postterminator $\left(1.0 \leq X_{\mathrm{VSO}} \leq 3.0\right)$.

\begin{tabular}{lrr|rr}
\hline & \multicolumn{2}{c|}{ Solar minimum } & \multicolumn{2}{c}{ Solar maximum } \\
\cline { 2 - 5 } Fitted & $-0.25-0.75$ & $1.0-3.0$ & $-0.25-0.75$ & $1.0-3.0$ \\
& $R_{\mathrm{V}}$ & $R_{\mathrm{V}}$ & $R_{\mathrm{V}}$ & $R_{\mathrm{V}}$ \\
\hline Upper & -0.011 & -0.002 & 0.013 & -0.005 \\
Mean & -0.005 & -0.002 & 0.012 & -0.005 \\
Median & -0.005 & -0.001 & 0.009 & -0.005 \\
Lower & -0.001 & -0.001 & 0.003 & -0.002 \\
\hline
\end{tabular}

\subsection{Mirror mode growth rate}

To enable a discussion of the growth rate of the MM waves, first the distribution of the event strengths along the magnetosheath flow direction needs to be investigated. In order to do that the data are split up into three bins in the direction perpendicular to the Venus-Sun line: $0 \leq R \leq 1.0$, $1.0 \leq R \leq 1.5$ and $1.5 \leq R \leq 2.5$. For each event the distance along the flow lines to the model bow shock (for either solar minimum or maximum, as described in Sect. 3) is also calculated, as shown by the cyan dotted arrows in Fig. 2 as an example.

First the distribution of $\mathcal{B}$ near the bow shock is studied, zooming in on the region $\pm 0.5 R_{\mathrm{V}}$ in $X_{\mathrm{VSO}}$ around the bow shock, as shown in the bottom panels of Fig. 5. It is clear that there is a broad range of $\mathcal{B}$ values in the freshly shocked plasma; there is not a single strength at which the MM waves are created. Indeed, this was also shown by Tátrallyay et al. (2008) in the Earth's magnetosheath, in their Fig. 3, where a range $3 \leq \Delta B \leq 10 \mathrm{nT}$ is observed just within the bow shock.

Tátrallyay et al. (2008) also used their data to estimate the growth rate of the MM waves, by a linear fit in log-space, and found a value of $\gamma=0.0022 \mathrm{~s}^{-1}$. Overall in the Earth's magnetosheath they find $0.001 \leq \gamma \leq 0.01$.

In order to investigate the MM growth rate at Venus, the data for the green population in Fig. 5, with a distance from the Venus-Sun line between $1.0 \leq R \leq 1.5$ is used, in which the highest occurrence rate is found and where the flow lines can be assumed to be almost parallel to $X_{\mathrm{VSO}}$. The lower quartile (cyan), mean (red), median (blue) and upper quartile (magenta) values are fitted, where the data are again binned in $0.25 R_{\mathrm{V}}$ bins along $X_{\mathrm{VSO}}$, as shown in Fig. 6. The estimated growth rates are shown in Table 2 .

Interestingly, the fits for solar minimum show a decaying MM population, after being created behind the bow shock. During solar maximum, however, all fits show a positive growth rate for the MM populations. Indeed, this agrees with the observations in Fig. 3, where the observational rate from squares A and B decreases going down the magnetosheath, whereas the observational rate from squares $\mathrm{C}$ and $\mathrm{D}$ increases. At solar minimum the MM waves are generated near the bow shock and decay, with a possible increase close to Venus due to draping of the magnetic field. At solar maximum the MM waves develop along the plasma flow in the magnetosheath.

This calls for a closer look at the distribution of the MM $\mathcal{B}$ near the bow shock. The top panels of Fig. 5 show the distribution of the strength $\mathcal{B}$ as a function of distance along the flow line to the model bow shock location, colour coded by the distance from the Venus-Sun line. The bottom panels show a zoom-in on $1 R_{\mathrm{V}}$ around the model bow shock location $X_{\mathrm{VSO}}=0$. It shows that for all data the sudden increase of MM waves near the bow shock is much more pronounced for solar minimum than for solar maximum. During solar maximum the bow shock is approximately $0.25 R_{\mathrm{V}}$ further out from Venus (see also e.g. Alexander and Russell, 1985), and the MM waves are on average weaker than for solar minimum. For the first few bins in Fig. 6 it is found that $\overline{\mathcal{B}} \approx 0.58 \pm 0.36$ for solar minimum, whereas $\overline{\mathcal{B}} \approx 0.39 \pm 0.19$ for solar maximum.

Behind Venus, i.e. behind the terminator, the MM waves decay in all cases, with decay rates, for solar maximum, which are approximately half the growth rate.

\section{Discussion}

The comparison of the statistical studies of MM waves at solar minimum and at solar maximum shows some expected results; however, some unexpected distributions are also found. The main difference between solar minimum and maximum for cycle is that the Sun radiates more UV, enhancing ionization of Venus's exosphere. Also, it should be noticed that the 2011-2012 solar maximum was very weak, with an exceptionally low proton density of the solar wind (Delva et al., 2015). It was found that the "undisturbed" solar wind has a density range of $0.5 \leq n_{\mathrm{p}} \leq 20 \mathrm{~cm}^{3}$ for solar minimum, whereas for solar maximum the range is more than a factor of 2 lower: $0.5 \leq n_{\mathrm{p}} \leq 8 \mathrm{~cm}^{3}$. The solar wind velocity is on average slightly lower for solar minimum, $\sim 300 \mathrm{~km} \mathrm{~s}^{-1}$, than for solar maximum $\sim 350 \mathrm{~km} \mathrm{~s}^{-1}$. The solar wind magnetic field does not significantly change, with a median value of $B_{\mathrm{sw}} \approx 9.88 \mathrm{nT}$ for solar minimum and $\approx 9.99 \mathrm{nT}$ for solar maximum.

At solar maximum the UV radiation of the Sun increases and thus there will be more ionization of the neutrals in Venus's exosphere. This is also the reason for the increase in ion cyclotron waves upstream of Venus's bow shock as shown by Delva et al. (2015). The increased ionization also causes the bow shock and ionosphere to move outward (Alexander and Russell, 1985; Shan et al., 2015), albeit Slavin et al. (1980) argue that charge exchange at low altitudes near the ionopause is causing the shock to move closer at solar minimum. 

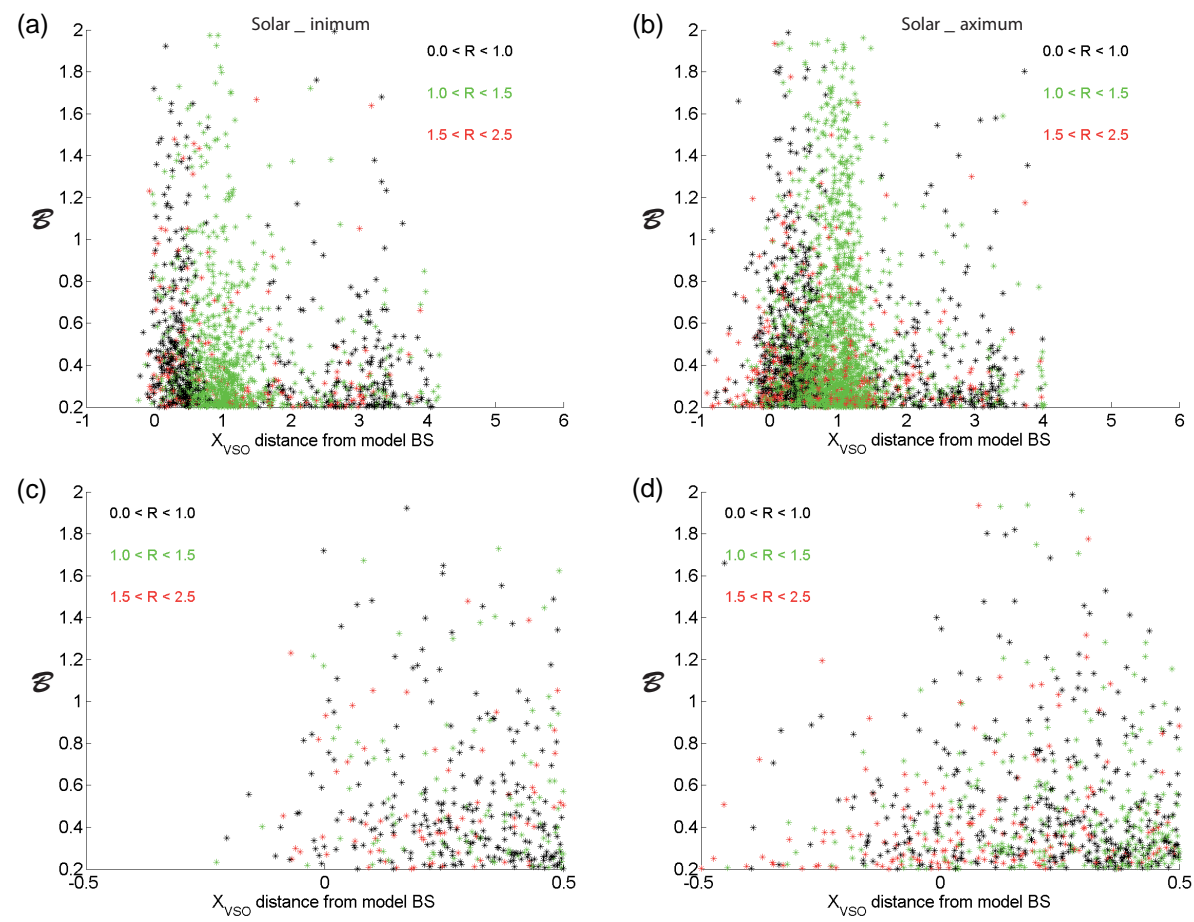

Figure 5. The distribution of the strength $\mathcal{B}$ of the MM waves as a function of distance to the model bow shock. The different colours indicate the different bins in $R$, the distance from the Venus-Sun line, as labelled in the panels. The top panels show the distribution for all events, the bottom panels are a zoom-in on $1 R_{\mathrm{V}}$ around the location of the model bow shock at $x_{\mathrm{VSO}}=0$.

The MM wave effect is to balance magnetic pressure $B^{2} / 2 \mu_{0}$ and plasma pressure $n_{i} k_{\mathrm{B}} T_{\perp, i}$, and the instability is driven by the temperature anisotropy of the ions (see Eq. 1). This means that the distribution of the MM waves with respect to $\mathcal{B}$ is most likely a reflection of the energy distribution of the ions in Venus's magnetosheath. Unfortunately, there are no papers discussing the plasma properties of Venus's magnetosheath for solar minimum and maximum. Also the cadence of the plasma instrument ASPERA (Barabash et al., 2007) is more than $3 \mathrm{~min}$, much too long to investigate the MM ion details, as the MM waves have a period between 4 and $15 \mathrm{~s}$.

The changes in bow shock for solar maximum, moving outward and thus increasing in size, and increased ionization by the solar UV radiation could, in principle, increase the number of MM waves generated behind the bow shock in Venus's magnetosheath. This is, however, not visible in Fig. 1. The first result of the comparison between solar minimum and maximum is that there are more MM waves found for solar maximum (a total of 1857 events) than for solar minimum (a total of 1637 events). The increased size of the bow shock and thus magnetosheath could be responsible for the increased observed number of MM events.

For solar minimum the fitted bow shock location is used from VEX measurements. For solar maximum such a determination from VEX data was not available, and therefore the model from Pioneer Venus data was used. The question may arise as to whether the solar maximum model is sufficiently accurate to use in order to determine the behaviour of the mirror mode waves in Venus's magnetosheath, as has been done in Figs. 3 and 6. Looking at the observations of MM waves as shown in Fig. 2, it is clear from both panels that the average location of the bow shock fits the data reasonably well, with a slightly larger discrepancy for solar maximum. Unfortunately, there are no error bars given for either of the bow shock fits.

This difference in bow shock location has no influence on the observational rates given in Fig. 3, but it could have consequences for the fits in Fig. 6. Figure 2 shows that in the region of interest for Fig. 6 (i.e. $1.0 \leq R \leq 1.5 R_{\mathrm{V}}$ ) there are only very few events that lie outside the model bow shock, and these points have not been taken into account in the determination of the growth rates of the MM waves.

Naturally, for a "perfect" fit, the distance to the observed bow shock would have to be determined, which is because of the great number of events unfeasible. This means that some of the distances can be incorrect. When the results from the observational rates in Fig. 3 are compared with the results of the growth rates in Fig. 6, it is clear that the two are in agreement.

Previous results by Génot et al. (2009) and Dimmock et al. (2015) have shown that the occurrence rate of MM waves in the Earth's magnetosheath is positively correlated to the Alfvén Mach number of the upstream solar wind. Figure 3 

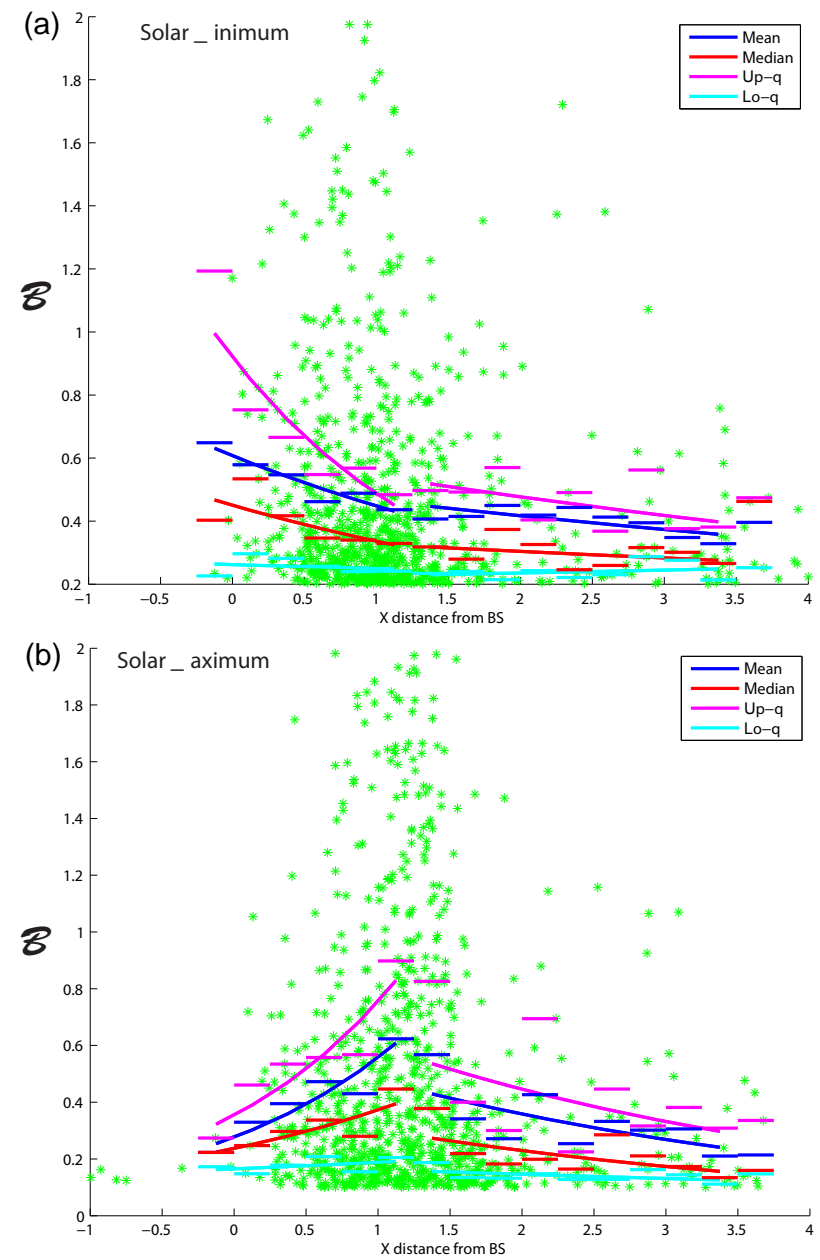

Figure 6. The distribution of the strength $\mathcal{B}$ of the $\mathrm{MM}$ waves as a function of distance to the model bow shock for $1.0 \leq R \leq 1.5$, the green population in Fig. 5. The coloured bars show the lower quartile (cyan), mean (red), median (blue), upper quartile (magenta) and maximum (black) values of each bin. The fits to these values are shown in the same colours, and the obtained growth rates are listed in Table 2.

shows, however, that although the number of events for solar maximum has increased slightly, the observational rate as defined in Eq. (4) does not particularly change. Indeed, taking into account the results by Delva et al. (2015) the decrease in average solar wind proton density by a factor of $\sim 2$ and the increase in average solar wind velocity by a factor of $\sim 1.2$ show that the average solar wind Alfvén Mach number changes by $1.2 / \sqrt{2} \approx 0.9$ from solar minimum to solar maximum. Therefore, a significant difference in the MM occurrence rate is not expected from this slight enhancement. The observational rate is overall the same but differently distributed over Venus's magnetosheath. This is most likely a result of the bow shock conditions for solar minimum and maximum being dissimilar (e.g. strength or thickness), which then energize the ions differently.
Not all MM waves have equal strength $\mathcal{B}$, as this depends on the available energy of the ions perpendicular to the magnetic field after being shocked by the bow shock crossing. Interestingly, it was found that just behind (i.e. the first three bins in Fig. 6) the bow shock, where freshly generated MM waves are expected, the average strength for solar minimum $\overline{\mathcal{B}} \approx 0.59 \pm 0.36$ (32 events) is higher than for solar maximum with $\overline{\mathcal{B}} \approx 0.32 \pm 0.22$ (23 events), and also the spread of the strengths is larger for solar minimum as indicated by the given standard deviation. The different average values listed here may or may not be significant. Because of the large standard deviation on these numbers one would be inclined to assume that there is no significance. However, this difference could also indicate that for solar minimum the energization of the ions in the ring distribution, through crossing the quasi-perpendicular bow shock, is stronger for solar minimum, and also that the variation of the bow shock strength is greater for solar minimum. There are no observational papers studying any possible differences for the bow shock for different solar activity conditions. It can also mean that the plasma conditions in the solar minimum magnetosheath are different from solar maximum. Unfortunately, there is also no study of Venus's magnetosheath plasma environments during solar minimum and maximum.

All MM waves were binned as a function of $\mathcal{B}$, with the result shown in Fig. 4. The binned data indicate an exponential fall-off in the number of MM waves with increasing $\mathcal{B}$. There seems to be a break in the slope near $\mathcal{B} \approx 1$. For the weak $\mathrm{MM}$ waves $(\mathcal{B} \leq 1.2)$ the slopes for solar minimum (maximum) are $a \approx-3.39 \pm 0.02(-3.04 \pm 0.03)$, whereas for strong MM waves $(\mathcal{B} \leq 0.8)$ the slopes are $a \approx-2.45 \pm$ $0.10(-1.82 \pm 0.01)$. This break can be created by the fact that the MM waves are observed during their growth and decay phase in $X_{\mathrm{VSO}}>0$; however, for $X_{\mathrm{VSO}}<0$ all MM waves are decaying whereby the number of "weak" MM waves observed can be increased.

Assuming that the MM waves grow and/or decay when they are transported through the magnetosheath, Tátrallyay et al. (2008) determined a growth rate by fitting $\Delta B$ of the MM waves as a function of flow time in the Earth's magnetosheath, finding an overall growth rate of $0.001 \leq \gamma \leq$ $0.01 \mathrm{~s}^{-1}$. In this current paper the MM waves located at distances from the Venus-Sun line between $1.0 \leq R \leq 1.5$ are used to obtain a growth rate; however, not the whole cloud of points is used, but the quantities are listed in Table 2 . For solar minimum all fits show negative values, indicating immediate decay of the MM waves after their generation behind the bow shock. Nevertheless, this does not exclude MM wave growth as clearly there are very strong events $\mathcal{B} \geq 1.2$ observed at farther distances from the bow shock, which can also be related to field line draping. For solar maximum, on the other hand, all fits show positive growth rates $0.003 \leq \gamma \leq 0.018 \mathrm{~s}^{-1}$, well within the range that Tátrallyay et al. (2008) found for the Earth's magnetosheath. Recently, Hoilijoki et al. (2016) used a 5-D Vlasov simulation (2-D 
space and 3-D velocity) to study MM waves in the Earth's magnetosheath. The obtained simulated growth rate for the MM waves was $0.002 \leq \gamma \leq 0.005 \mathrm{~s}^{-1}$, which does not completely cover the ranges estimated from observations in this paper and in Tátrallyay et al. (2008).

The plasma transport time across the magnetosheath can be estimated as $t_{\mathrm{tr}} \approx 0.5 R_{\mathrm{V}} / v_{\mathrm{pl}} \approx 30 \mathrm{~s}$, with $R_{\mathrm{V}}=6052 \mathrm{~km}$ and $v_{\mathrm{pl}}=100 \mathrm{~km} \mathrm{~s}^{-1}$ the nominal flow velocity in the magnetosheath (e.g. Guicking et al., 2010). With the maximum growth rate as determined above $t_{\text {tr }}$ relates to half an $e$ folding time. However, in the region $1.0 \leq R \leq 1.5$, the wave growth seems to extend over five bins in Fig. 6, which is $\sim 1.25 R_{\mathrm{V}}$ and thus a maximum of $\sim 1.25 e$-folding times. There is time for the MM waves to evolve while they move toward the terminator. After the MM waves cross the terminator, where the pick-up density is highest (Delva et al., 2015) and the magnetic field starts to diverge, the magnetosheath becomes MM stable and the waves start to decay.

When the MM waves are transported down the magnetosheath, they will eventually enter a MM-stable region and will have to start to decay. Indeed, Figs. 5 and 6 show that $\mathcal{B}$ falls off. Table 2 shows the determined decay rates: $-0.009 \leq \gamma \leq-0.001 \mathrm{~s}^{-1}$ for $1.0 \leq R \leq 1.5$. There are no quantitative models for the decay of MM waves. Joy et al. (2006) assume a stochastic leaking of ions out of the magnetic bottle (using the model by Constantinescu, 2002), thereby reducing the plasma pressure and the magnetic tension then starts to straighten the field lines. However, there is no given decay rate for this model.

\section{Conclusions}

Comparing the MM characteristics in Venus's magnetosheath between solar minimum and maximum conditions, the following is found:

- There are slightly more MM events $(\sim 14 \%)$ for solar maximum than for solar minimum.

- The observational rate for both solar conditions is the same because of the interplay of lower solar wind density and higher solar wind velocity during solar maximum than during solar minimum. ${ }^{1}$

- The distribution of the number of MM waves as a function of the strength $\mathcal{B}$ is exponential with approximately the same coefficient for both solar conditions for "weak" MM waves (i.e. $\mathcal{B} \leq 1.2$ ). There is a less steep exponential for "strong" $M M$ waves (i.e. $\mathcal{B} \geq 0.8$ ) with significant differences in the exponential for solar minimum and maximum.

\footnotetext{
${ }^{1}$ Cycle 24 is known to have a very weak solar maximum and thus may not be representative of more "regular" maxima.
}

- Freshly created MM waves behind the bow shock are on average stronger for solar minimum than for solar maximum.

- For solar minimum the general trend for MM waves is to decay; for solar maximum all MM waves grow, between the bow shock and the terminator.

- The estimated growth rates for the MM waves agree well with those found for the Earth's magnetosheath.

\section{Data availability}

The Venus Express magnetometer data is available from ESA's Planetary Science Archive (ESA, 2014, http://www. cosmos.esa.int/web/psa/venus-express).

Acknowledgements. The work by Daniel Schmid was supported by the Austrian Science Fund FWF under grant P25257-N27. Portions of this work were performed at the Jet Propulsion Laboratory, California Institute of Technology under contract with NASA.

The topical editor, Y. Miyoshi, thanks G. Erdös and one anonymous referee for help in evaluating this paper.

\section{References}

Alexander, C. J. and Russell, C. T.: Solar cycle dependende of the location of the Venus bow shock, Geophys. Res. Lett., 12, 369371, 1985.

Barabash, S., Sauvaud, J.-A., Gunell, H., Andersson, H., Grigoriev, A., Brinkfeldt, K., Holmström, M., Lundin, R., Yamauchi, M., Asamura, K., Baumjohann, W., Zhang, T., Coates, A., Linder, D., Kataria, D., Curtis, C., Hsieh, K., Sandel, B., Fedorov, A., Mazelle, C., Thocaven, J.-J., Grande, M., Koskinen, H. E., Kallio, E., Säles, T., Riihela, P., Kozyra, J., Krupp, N., Woch, J., Luhmann, J., McKenna-Lawlor, S., Orsini, S., Cerulli-Irelli, R., Mura, M., Milillo, M., Maggi, M., Roelof, E., Brandt, P., Russell, C., Szego, K., Winningham, J., Frahm, R., Scherrer, J., Sharber, J., Wurz, P., and Bochsler, P.: The analyser of space plasmas and energetic atoms (ASPERA-4) for the Venus Express mission, Planet. Space Sci., 55, 1772-1792, doi:10.1016/j.pss.2007.01.014, 2007.

Baumjohann, W., Treumann, R. A., Georgescu, E., Haerendel, G., Fornaçon, K.-H., and Auster, U.: Waveform and packet structure of lion roars, Ann. Geophys., 17, 1528-1534, doi:10.1007/s00585-999-1528-9, 1999.

Bavassano Cattaneo, M. B., Basile, C., Moreno, G., and Richardson, J. D.: Evolution of mirror structures in the magnetosheath of Saturn from the bow shock to the magnetopause, J. Geophys. Res., 103, 11961-11972, 1998.

Constantinescu, O. D.: Self-consistent model of mirror structures, J. Atm. Solar Terr. Phys., 64, 645-649, 2002.

Crooker, N. U. and Siscoe, G. L.: A Mechanism for Pressure Anisotropy and Mirror Instability in the Dayside Magnetosheath, J. Geophys. Res., 82, 185-186, doi:10.1029/JA082i001p00185, 1977. 
Czaykowska, A., Bauer, T. M., Treumann, R. A., and Baumjohann, W.: Magnetic field fluctuations across the Earth's bow shock, Ann. Geophys., 19, 275-287, doi:10.5194/angeo-19-275-2001, 2001.

Delva, M., Zhang, T. L., Volwerk, M., Vörös, Z., and Pope, S. A.: Proton cyclotron waves in the solar wind at Venus, Geophys. Res. Lett., 113, E00B06, doi:10.1029/2008JE003148, 2008.

Delva, M., Bertucci, C., Volwerk, M., Lundin, R., Mazelle, C., and Romanelli, N.: Upstream proton cyclotron waves at Venus near solar maximum, J. Geophys. Res., 120, 344-354, doi:10.1002/2014JA020318, 2015.

Dimmock, A. P., Osmane, A., Pulkkinen, T. I., and Nykyri, K.: A statistical study of the dawn-dusk asymmetry of ion temperature anisotropy and mirror mode occurrencein the terrestrial daysidemagnetosheath using THEMIS data, J. Geophys. Res., 120, 5489-5503, doi:10.1002/2015JA021192, 2015.

Du, J., Wang, C., Zhang, T. L., and Kallio, E.: Asymmetries of the magnetic field line draping shape around Venus, J. Geophys. Res., 118, 6915-6920, doi:10.1002/2013JA019127, 2013.

Erdös, G. and Balogh, A.: Statistical properties of mirror mode structures observed by Ulysses in the magnetosheath of Jupiter, J. Geophys. Res., 101, 1-12, 1993.

ESA: Venus Express magnetometer data, European Space Agency, available at: http://www.cosmos.esa.int/web/psa/venus-express, 2014.

Gary, S. P.: Electromagnetic ion/ion instabilities and their consequences in space plasmas: A review, Space Sci. Rev., 56, 373415,1991 .

Génot, V., Budnik, E., Jacquey, C., Dandouras, I., and Lucek, E.: Mirror modes observed with Cluster in the Earth's magnetosheath: statistical study and IMF/solar wind dependence, Adv. Geophys., 14, 1-22, 2009.

Glassmeier, K. H., Motschmann, U., Mazalle, C., Neubauer, F. M., Sauer, K., Fuselier, S. A., and Acuña, M. H.: Mirror modes and fast magnetoaucoustic waves near the magnetic pileup boundary of comet P/Halley, J. Geophys. Res., 98, 20955-20964, 1993.

Guicking, L., Glassmeier, K.-H., Auster, H.-U., Delva, M., Motschmann, U., Narita, Y., and Zhang, T. L.: Low-frequency magnetic field fluctuations in Venus' solar wind interaction region: Venus Express observations, Ann. Geophys., 28, 951-967, doi:10.5194/angeo-28-951-2010, 2010.

Hasegawa, A.: Drift mirror instability in the magnetosphere, Phys. Fluids, 12, 2642-2650, 1969.

Hoilijoki, S., Palmroth, M., Walsh, B. M., Pfau-Kepmf, Y., von Alfthan, S., Ganse, U., Hannuksela, O., and Vainio, R.: Mirror modes in the Earth's magnetosheath: Results from a global hybrid-Vlasov simulation, J. Geophys. Res., 121, 4191-4204, doi:10.1002/2015JA022026, 2016.

Joy, S. P., Kivelson, M. G., Walker, R. J., Khurana, K. K., and Russell, C. T.: Mirror mode structures in the Jovian magnetosheath, J. Geophys. Res., 111, A12212, doi:10.1029/2006JA011985, 2006.

Lucek, E. A., Dunlop, M. W., Balogh, A., Cargill, P., Baumjohann, W., Georgescu, E., Haerendel, G., and Fornacon, G.-H.: Identification of magnetosheath mirror modes in Equator-S magnetic field data, Ann. Geophys., 17, 1560-1573, doi:10.1007/s00585999-1560-9, 1999.
Price, C. P., Swift, W., and Lee, L.-C.: Numerical Simulation of Nonoscillatory Mirror Waves at the Earth's Magnetosheath, J. Geophys. Res., 91, 101-112, 1986.

Remya, B., Tsurutani, B. T., Reddy, R. V., Lakhina, G. S., Falkowski, B. J., Echer, E., and Glassmeier, K.-H.: Large amplitude circularly polarized, compressive, obliquely propagating electromagnetic proton cyclotron waves throughout the Earth's magnetosheath: low plasma $\beta$ conditions, Astrophys. J., 93, 6 pp., doi:10.1088/0004-637X/793/1/6, 2014.

Russell, C. T., Chou, E., Luhmann, J. G., Brace, P. G. L. H., and Hoegy, W. R.: Solar and interplanetary control of the location of the Venus bow shock, J. Geophys. Res., 93, 5461-5469, 1988.

Schmid, D., Volwerk, M., Plaschke, F., Vörös, Z., Zhang, T. L., Baumjohann, W., and Narita, Y.: Mirror mode structures near Venus and Comet P/Halley, Ann. Geophys., 32, 651-657, doi:10.5194/angeo-32-651-2014, 2014.

Shan, L. C., Lu, Q. M., Mazelle, C., Huang, C., Zhang, T. L., Wu, M. Y., Gao, X. L., and Wang, S.: The shape of the Venusian bow shock at solar minimum and maximum: Revisit based on VEX observations, Planet. Space Sci., 109-110, 32-37, doi:10.1016/j.pss.2015.01.004, 2015.

Slavin, J. A., Elphic, R. C., Russell, C. T., Scarf, F. L., Wolfe, J. H., Mihalov, J. D., Intrilligator, D. S., Brace, L. H., Taylor, Jr., H. A., and Daniell, Jr., R. E.: The solar wind interaction with Venus: Pioneer Venus observations of bow shock location and structure, J. Geophys. Res., 85, 7625-7641, 1980.

Soucek, J., Escoubet, C. P., and Grison, B.: Magnetosheath plasma stability and ULF wave occurrence as a function of location in the magnetosheath and upstream bow shock parameters, J. Geophys. Res., 120, 2838-2850, doi:10.1002/2015JA021087, 2015.

Svedhem, H., Titov, D. V., McCoy, D., Lebreton, J.-P., Barabash, S., Bertaux, J.-L., Drossart, P., Formisano, V., Häusler, B., Korablev, O., Markiewicz, W. J., Nevejans, D., Pätzold, M., Piccioni, G., Zhang, T. L., Taylor, F. W., Lellouch, E., Koschny, D., Witasse, O., Eggel, H., Warhaut, M., Accomazzo, A., Rodriguez-Canabal, J., Fabrega, J., Schirmann, T., Clochet, A., and Coradini, M.: Venus Express: The first European mission to Venus, Planet. Space Sci., 55, 1636-1652, doi:10.1016/.pss.2007.01.013, 2007.

Tátrallyay, M., Erdös, G., Balogh, A., and Dandouras, I.: The eveloution of mirror type magnetic fluctuations in the magnetosheath based on multipoint observations, Adv. Space Res., 41, 15371544, doi:10.1016/j.asr.2007.03.039, 2008.

Tsurutani, B. T., Smith, E. J., Anderson, R. R., Ogilvie, K. W., Scudder, J. D., Baker, D. N., and Bame, S. J.: Lion roars and nonoscillatory drift mirror waves in the magnetosheath, J. Geophys. Res., 87, 6060-6072, 1982.

Tsurutani, B. T., Arballo, J. K., Zhou, X.-Y., Galvan, C., and Chao, J. K.: Electromagnetic electron and proton cyclotron waves in geospace: A Cassini snaphot, COSPAR Coll. Ser., 12, 97-125, 2002.

Tsurutani, B. T., Lakhina, G. S., Verkholyadova, O. P., Echer, E., Guarnieri, F. L., Narita, Y., and Constantinescu, D. O.: Magnetosheath and heliosheath mirror mode structures, interplanetary magnetic decreases, and linear magnetic decreases: Differences and distinguishing features, J. Geophys. Res., 116, A02103, doi:10.1029/2010JA015913, 2011.

Volwerk, M., Zhang, T. L., Delva, M., Vörös, Z., Baumjohann, W., and Glassmeier, K.-H.: First identification of mirror mode waves 
in Venus' magnetosheath?, Geophys. Res. Lett, 35, L12204, doi:10.1029/2008GL033621, 2008a.

Volwerk, M., Zhang, T. L., Delva, M., Vörös, Z., Baumjohann, W., and Glassmeier, K.-H.: Mirror-mode-like structures in Venus' induced magnetosphere, J. Geophys. Res., 113, E00B16, doi:10.1029/2008JE003154, 2008b.

Volwerk, M., Glassmeier, K.-H., Delva, M., Schmid, D., Koenders, C., Richter, I., and Szegö, K.: A comparison between VEGA 1, 2 and Giotto flybys of comet 1P/Halley: implications for Rosetta, Ann. Geophys., 32, 1441-1453, doi:10.5194/angeo-321441-2014, 2014.

Zhang, T. L., Baumjohann, W., Delva, M., Auster, H.-U., Balogh, A., Russell, C. T., Barabash, S., Balikhin, M., Berghofer, G., Biernat, H. K., Lammer, H., Lichtenegger, H., Magnes, W., Nakamura, R., Penz, T., Schwingenschuh, K., Vörös, Z., Zambelli, W., Fornacon, K.-H., Glassmeier, K.-H., Richter, I., Carr, C., Kudela, K., Shi, J. K., Zhao, H., Motschmann, U., and Lebreton, J.-P.: Magnetic field investigation of the Venus plasma environment: Expected new results, Planet. Space Sci., 54, 13361343, doi:10.1016/j.pss.2006.04.018, 2006.
Zhang, T. L., Delva, M., Baumjohann, W., Volwerk, M., Russell, C., Barabash, S., Balikhin, M., Pope, S., Glassmeier, K.-H., Kudela, K., Wang, C., Vörös, Z., and Zambelli, W.: Initial Venus express magnetic field observations of the Venus bow shock location at solar minimum, Planet. Space Sci., 56, 785-789, 2008a.

Zhang, T. L., Russell, C. T., Baumjohann, W., Jian, L. K., Balikhin, M. A., Cao, J. B., Wang, C., Blanco-Cano, X., Glassmeier, K.-H., Zambelli, W., Volwerk, M., Delva, M., and Vörös, Z.: Characteristic size and shape of the mirror mode structures in the solar wind at $0.72 \mathrm{AU}$, Geophys. Res. Lett., 35, L10106, doi:10.1029/2008GL033793, 2008b.

Zhang, T. L., Baumjohann, W., Du, J., Nakamura, R., Jarvinen, R., Kallio, E., Du, A. M., Balikhin, M., Luhmann, J. G., and Russell, C. T.: Hemispheric asymmetry of the magnetic field wrapping pattern in the Venusian magnetotail, Geophys. Res. Lett., 37, L14202, doi:10.1029/2010GL044020, 2010. 